Check for updates

Cite this: RSC Adv., 2017, 7, 40270

\title{
Biological phosphorylated molecules participate in the biomimetic and biological synthesis of cadmium sulphide quantum dots by promoting $\mathrm{H}_{2} \mathrm{~S}$ release from cellular thiols $\dagger$
}

\author{
F. A. Venegas, $\dot{t}^{a}$ L. A. Saona, ${ }^{\text {ab }}$ J. P. Monrás, ${ }^{a}$ N. Órdenes-Aenishanslins, ${ }^{\text {ab }}$ \\ M. F. Giordana, ${ }^{c}$ G. Ulloa, ${ }^{\text {ab }}$ B. Collao, ${ }^{a}$ D. Bravo ${ }^{d}$ and J. M. Pérez-Donoso (D) *a
}

Developing methods with a low environmental impact for nanoparticle synthesis remains one of the greatest challenges in nanotechnology. In this context, biomimetic and biological methods have emerged as green chemistry alternatives, and also contribute to our understanding of how nanomaterials interact with cellular molecules. Here, we report a phosphate-dependent biomimetic method to synthesize of cadmium sulphide (CdS) QDs at low temperatures, physiological $\mathrm{pH}$ and aerobic conditions, using $\mathrm{CdCl}_{2}$ and thiols (L-cysteine, glutathione or mercaptosuccinic acid). Inorganic phosphate (Pi) and cellular phosphorylated intermediates such as adenosine monophosphate, glucose-6-phosphate, glycerol-2-phosphate and fructose-1,6-biphosphate, can trigger CdS QDs synthesis. The produced QDs are cubic phase nanocrystals with a tunable fluorescence $(450-700 \mathrm{~nm})$, small size $(4-12 \mathrm{~nm})$, and are composed of thiols and Pi. In $\mathrm{CdS}$ synthesis, the importance of the phosphate is related to its capacity to release $\mathrm{H}_{2} \mathrm{~S}$ from thiols, a phenomenon associated with its base-properties. Based on the biomimetic method, we developed a $\mathrm{Pi}$ based procedure to synthesize CdS QDs in Escherichia coli. As in the biomimetic procedure, Pi favors QDs-biosynthesis not only by mediating biological generation of $\mathrm{H}_{2} \mathrm{~S}$, but also by improving $\mathrm{Cd}^{2+}$ cellular uptake. A role for phosphates in the cellular interaction and green synthesis of metal QDs is discussed.

Received 28th March 2017

Accepted 8th August 2017

DOI: $10.1039 / \mathrm{c} 7 \mathrm{ra03578k}$

rsc.li/rsc-advances diodes, ${ }^{3}$ in vitro and in vivo labelling of tumours or proteins ${ }^{4-8}$ and the analytical detection of cations., ${ }^{9,10}$

Cadmium sulphide (CdS) nanocrystals are one of the most studied QDs and during the last years numerous colloidal methods for CdS QDs synthesis with improved properties have been developed. However, these protocols can be toxic as they use organic solvents, and also expensive, as they require high temperatures (more than $200^{\circ} \mathrm{C}$ ), inert environments (nitrogen or argon) and expensive ligands.

To overcome these limitations, milder protocols for synthesizing water soluble-CdS QDs that use low temperatures, aerobic conditions, and thiols as capping agents and/or the sulphur source ${ }^{11,12}$ were developed. Nanoparticles produced by these methods show improved properties that favour their use in different applications, particularly those related with biological systems. In general, these "green" protocols using mild conditions and biomolecules are called "biomimetic".

Knowledge derived from biomimetic methods contributed to developing biological methods for the synthesis of NPs (biosynthesis) in cells (eukaryotic or prokaryotic) or viruses. ${ }^{13,14}$ In recent years, our laboratory developed a biomimetic method to synthesize CdTe NPs using the cellular thiol glutathione (GSH) ${ }^{15}$ which was later used to biosynthesize CdTe NPs in Escherichia coli overproducing GSH. ${ }^{16}$ 
In the present work, we explored a novel, simple and economic "green" protocol to synthesize CdS NPs based on the source of phosphate and thiols. Inorganic phosphate (Pi) and several biological phosphorylated molecules such as adenosine monophosphate (AMP), glucose-6-phosphate (G6P), glycerol-2phosphate (G2P) and fructose-1,6-biphosphate (F1,6BP) promoted the chemical synthesis of water soluble-CdS QDs at physiological temperature and aerobiosis. Further, in E. coli Pi stimulated the intracellular biosynthesis of CdS QDs, which was accompanied by increased $\mathrm{Cd}^{2+}$-uptake and $\mathrm{H}_{2} \mathrm{~S}$ generation.

\section{Experimental section}

\section{Reagents}

All reagents used in this work were purchased from SigmaAldrich and used without further purifications. Ingredients for bacterial growth media (LB, lysogeny broth) were purchased from Difco.

\section{Biomimetic synthesis of CdS QDs}

For biomimetic tests, $1 \mathrm{~mL}$ of each solution was prepared in $1.5 \mathrm{~mL}$ microcentrifuge tubes using the following compounds: cadmium chloride $\left(\mathrm{CdCl}_{2}\right)$, glutathione (GSH), L-cysteine (CYS), mercaptosuccinic acid (MSA), dibasic potassium phosphate $\left(\mathrm{K}_{2} \mathrm{HPO}_{4}\right)$, monobasic potassium phosphate $\left(\mathrm{KH}_{2} \mathrm{PO}_{4}\right)$, phosphate buffer $\mathrm{pH} 7.4$ (in this work as $\mathrm{PB}: \mathrm{KH}_{2} \mathrm{PO}_{4} / \mathrm{K}_{2} \mathrm{HPO}_{4}$ ), tribasic dihydrate sodium citrate (citrate), MOPS pH 7.4, Tris$\mathrm{HCl} \mathrm{pH} 7.4$, HEPES pH 7.4, triethylamine $\left(\mathrm{Et}_{3} \mathrm{~N}\right)$ or the organic phosphorylated molecules (G6P, G2P, F1,6BP, AMP, ADP or ATP). Incubation time of days or hours were required depending on temperatures used for synthesis $\left(37\right.$ or $80{ }^{\circ} \mathrm{C}$, respectively).

The qualitative fluorescence emission over time was evaluated exposing microcentrifuge tubes directly on a (UV) transilluminator $\left(\lambda_{\text {exc }} 365 \mathrm{~nm}\right)$.

To remove the remaining metal salts and reagents from synthesis solutions, each reaction was filtered using Amicon filters (ultra- 4 centrifugal filter units of Millipore), with a pore size of $0.3-0.5 \mathrm{~nm}$ and centrifuged at $98.3 \mathrm{~Hz}$ until a $\sim 200 \mu \mathrm{L}$ solution was obtained. Each solution was washed twice with milliQ water and used for characterization experiments (see below).

A standard biomimetic synthesis protocol was composed of $5 \mathrm{mM}$ MSA, $54.5 \mu \mathrm{M} \mathrm{CdCl}_{2}$ and $10 \mathrm{mM}$ of a phosphate source (Pi or a phosphorylated molecule), at $37^{\circ} \mathrm{C}$.

\section{Absorbance and fluorescence spectroscopy}

Spectroscopic characteristics of synthesized NPs were determined using a spectrophotometer/spectrofluorimeter Synergy H1 (Biotek). UV-Vis spectra of CdS nanoparticles were used to determine the band gap $\left(E_{\mathrm{bg}}\right)$ by the Tauc relation. ${ }^{17}$ For quantum yield (QY) determination, 4 to 5 samples were prepared with different absorbance values between 0.01 and 0.1 at an excitation wavelength of $360 \mathrm{~nm}$. The procedure was applied for green and red purified NPs dissolved in water and for fluorescein diacetate in ethanol $(\mathrm{QY}=0.9)$. Fluorescence spectra were recorded by obtaining the integrated fluorescence intensity (IFI). IFI was plotted versus the absorbance of the solution. The slope for each curve $(m)$ and the refractive index of the solvent $(n)$ (water: 1.333 , and ethanol: 1.335$)$ were used to calculate NPs QY considering fluorescein diacetate as a reference, using the following equation: $\mathrm{QY}_{\mathrm{NPs}}=\mathrm{QY}_{\mathrm{R}}\left[m_{\mathrm{NPs}} / m_{\mathrm{R}}\right]$ $\left[n_{\mathrm{NPs}}^{2} / n_{\mathrm{R}}^{2}\right]$.

\section{Fourier transform infrared spectroscopy (FT-IR)}

Samples were lyophilized and NPs powder in KBr pellets were measured in a solid FT-IR spectrometer between 4000 and $400 \mathrm{~cm}^{-1}$ (Spectrum Two, Perkin Elmer). Appropriate controls (thiols, phosphate, $\mathrm{CdCl}_{2}$, phosphorylated molecules, among others) were measured in the same way as described above.

\section{Dynamic light scattering (DLS)}

DLS measurements of NPs samples were carried out in 4 optical path cuvettes using a Zetasizer Nano ZS (Malvern Instrument Limited, UK).

\section{Transmission electron microscopy (TEM) and high resolution transmission electron microscopy (HR-TEM)}

The size and crystalline structure of purified NPs were determined using a TEM FEI Tecnai F20 G2 with a field emission gun operated at $200 \mathrm{kV}$. QDs solutions were dispersed ultrasonically and some drops of the suspension were deposited on an ultrathin holey carbon copper grid.

\section{CdS QDs biosynthesis}

A single colony of $E$. coli BW25113 was grown overnight in liquid LB medium with a constant agitation $(4.17 \mathrm{~Hz})$ at $37^{\circ} \mathrm{C}$. A $1 / 100$ dilution from the saturated culture was prepared in LB medium $(100 \mathrm{~mL})$ and incubated at $37^{\circ} \mathrm{C}(4.17 \mathrm{~Hz})$ to an optical density at $600 \mathrm{~nm}\left(\mathrm{DO}_{600}\right)$ between 1.5 and 2.0. Then, cells were centrifuged at $123 \mathrm{~Hz}$ during $10 \mathrm{~min}$, the supernatant was discarded, and the cellular pellet was washed twice with $10 \mathrm{~mL}$ of $500 \mathrm{mM}$ Tris- $\mathrm{HCl} \mathrm{pH}$ 7.4. The resulting culture was divided in different fractions, supplemented with $\mathrm{CdCl}_{2}(327.2 \mu \mathrm{M}$ or $60 \mu \mathrm{g} \mathrm{mL} \mathrm{m}^{-1}$ final concentration) and/or $\mathrm{Pi}$ and incubated at $37{ }^{\circ} \mathrm{C}(4.17 \mathrm{~Hz})$. Pi concentrations of $0 ; 1 ; 2.5 ; 5 ; 7.5 ; 10 ; 20 ; 30$; 40 and $50 \mathrm{mM}$ were used. To evaluate CdS QDs biosynthesis over time, fluorescence emission was evaluated by exposing cell pellets to UV light $\left(\lambda_{\text {exc }} 365 \mathrm{~nm}\right)$, as previously described by Monrás et al., 2013.

\section{Intracellular Cd quantification by flame atomic absorption spectroscopy (FAAS)}

Bacterial pellets from $10 \mathrm{~mL}$ cultures were degraded using $7 \mathrm{~N}$ $\mathrm{HNO}_{3}$ for $16 \mathrm{~h}$ at $37^{\circ} \mathrm{C}$. One hundred microliter samples were diluted to $4 \mathrm{~mL}$ in milliQ water and measured in a flame emission spectrometer AA6200 (Shimadzu). A Cd calibration curve was built using a commercial standard (Sigma Aldrich). Intracellular $\mathrm{Cd}$ values were normalized by protein concentration. Proteins were quantified by the Bradford method (Bradford, 1976). 


\section{$\mathrm{H}_{2} \mathrm{~S}$ release measurement}

A white filter paper (paper matrix) was moistened with a 5\% lead acetate solution, dried $\left(60^{\circ} \mathrm{C}\right)$ and then fixed to the lid of the tubes under the different conditions (in three or more independent tubes). PbS formation was visualized by a change in the colour, according to the lead acetate method. ${ }^{18}$ Pictures for all different paper matrixes were taken and the results were plotted in duplicates considering the following: for each image a rectangular zone (with the same number of pixels) was selected, within these zones, pixel intensity was quantified considering a grayscale. A value between 0 and 255 was assigned for each pixel, where 0 represents black and 255 white. The final values were plotted as the inverse of the intensity of the total selected pixels to facilitate interpretation. Analyses were made using the ImageJ software (http://imagej.nih.gov/ij/).

\section{Statistical analysis}

Statistically significant differences $(P<0.05)$ were determined using the Test student ( $t$-test) with the GraphPad Prims v5.0 software.

\section{Results and discussion}

\section{Phosphate is required to trigger the biomimetic synthesis of CdS QDs}

The "quantum confinement effect" establishes a relationship between the distances in the energy levels in which electrons are confined in the nanocrystals ${ }^{\mathbf{1 9}}$ of QDs. Because of this effect, small QDs absorb light and emit fluorescence at lower wavelengths than bigger QDs and show differences in the emission colours. This characteristic was used as a screening for potential NPs synthesis.

The fluorescence of solutions composed by a thiol [L-cysteine (CYS), GSH or mercaptosuccinic acid (MSA)], a cadmium salt and PBS pH 7.0 evolved over time from green-yellow to orangered (data not shown). To explore more conditions and establish a simple biomimetic system for QDs synthesis, different Cd concentrations, two temperatures, and only $\mathrm{Pi}$ in the form of $\mathrm{K}_{2} \mathrm{HPO}_{4}$ (component of PBS with intrinsic pH between 8.9 and 9.5) and MSA (the fastest of the three thiols to produce QDs) were used as substrates. As shown in Fig. 1, the tested conditions had different effects over the fluorescence of the solutions: Pi generated fluorescent solutions that changed their emission colours over time from green to red (Fig. 1A), Pi concentration modulated the colour of the solution at a fixed time (Fig. 1B), the amount of $\mathrm{Cd}$ regulated the fluorescence colour (Fig. 1C), and temperature modulated the time at which solutions became fluorescent; at low temperatures this was observed within days $\left(37^{\circ} \mathrm{C}\right.$ or even $15{ }^{\circ} \mathrm{C}$, data not shown), while at temperatures between 80 and $100{ }^{\circ} \mathrm{C}$ within hours (Fig. 1C).

To demonstrate that the fluorescent solutions contained QDs, nanoparticulated material from two different fluorescent solutions (orange-red and blue-green) was purified and characterized. Both solutions showed a maximal absorbance between 380 and $360 \mathrm{~nm}$ and the typical surface plasmon absorbance shoulder (below $400 \mathrm{~nm}$; Fig. 2A) described for
A
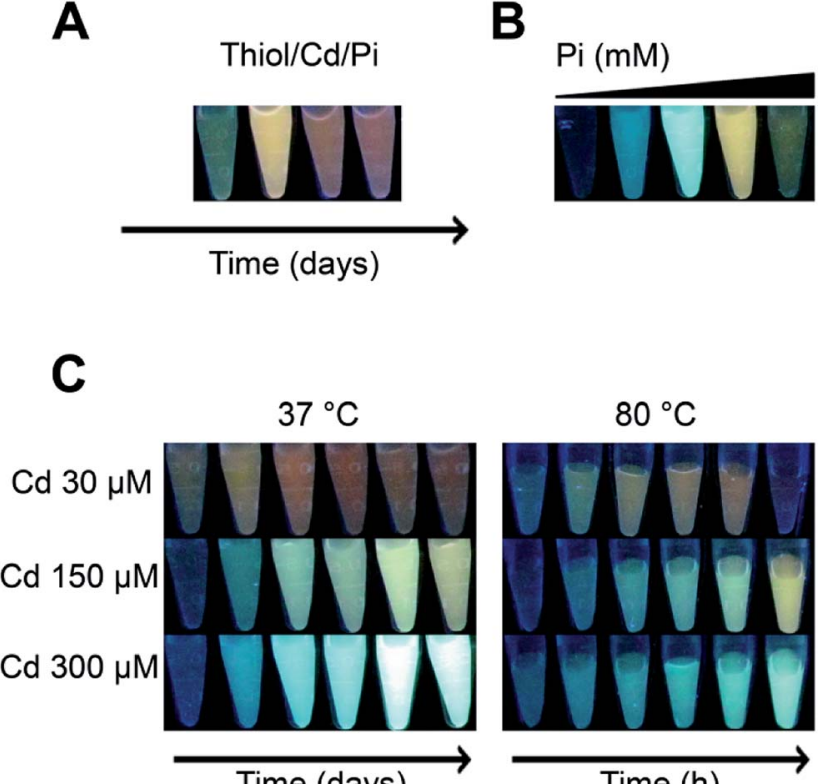

Time (days)

Fig. 1 Effect of $\mathrm{Pi}, \mathrm{Cd}$ and temperature on the fluorescence of biomimetic CdS QDs. (A) Reaction tubes containing $10 \mathrm{mM} \mathrm{Pi}, 54.5 \mu \mathrm{M}$ $\left(10 \mu \mathrm{g} \mathrm{mL}^{-1}\right) \mathrm{CdCl}_{2}$ and $5 \mathrm{mM} \mathrm{GSH}$ were incubated at $37^{\circ} \mathrm{C}$ from 1 to 4 days. In (B) solutions containing $\mathrm{CdCl}_{2} 150 \mu \mathrm{M}, \mathrm{MSA} 5 \mathrm{mM}$ and $\mathrm{Pi}$ 0, 7.5, 10, 20 or $75 \mathrm{mM}$, were incubated at $37^{\circ} \mathrm{C}$ during 6 days. (C) Solutions containing Pi $10 \mathrm{mM}, \mathrm{CdCl}_{2} 30,150$ or $300 \mu \mathrm{M}$, and MSA $5 \mathrm{mM}$, were incubated at $37^{\circ} \mathrm{C}$ ( 1 to 6 days) or at $80^{\circ} \mathrm{C}(0.5,1,1.5,2,2.5,3$ and $4 \mathrm{~h}$ ).

water soluble CdS QDs. Further, blue-green solutions emitted at $450 \mathrm{~nm}$ and orange-red at $560 \mathrm{~nm}$ (Fig. 2B), as previously reported for CdS QDs. ${ }^{20}$ The observed differences in absorbance and fluorescence between both solutions are related to NP size. The average size of orange-red and blue-green solutions was 7.5 and $3.5 \mathrm{~nm}$, respectively (Fig. 2C and D). A wide size distribution was determined in both QDs synthesis, a result that agrees with the observed broad emission spectra. In addition, DLS analysis
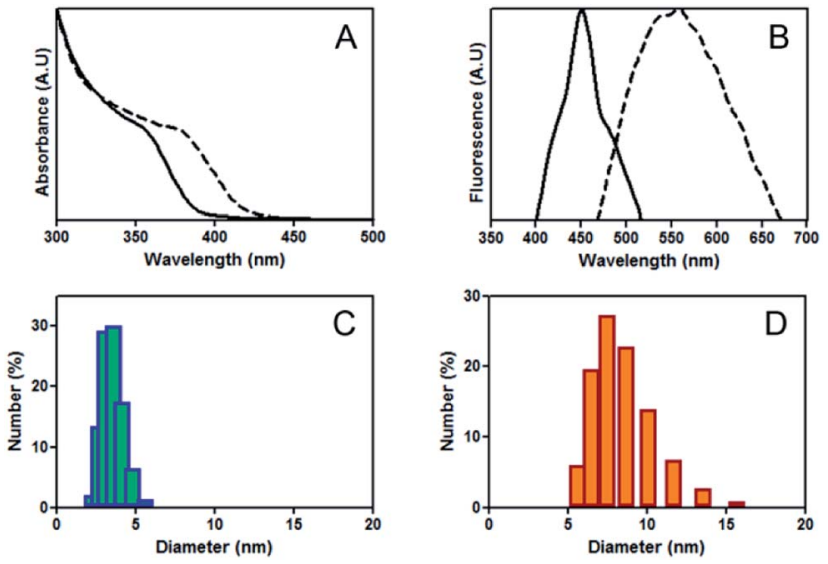

Fig. 2 Characterization of CdS QDs. (A) Absorbance and (B) fluorescence spectra of blue-green NPs (continuous line) and orange-red (dashed line). DLS determination of blue-green (C) and orange-red NPs (D); number\% corresponds to volume\%. Complete DLS analysis in Fig. S1. $\dagger$ 
shows higher size peaks (near $230 \mathrm{~nm}$ ), most probably corresponding to NPs aggregates (Fig. S1 $\dagger$ ). QDs self-aggregation is consequence of non-covalent interactions between capping materials, a phenomenon that is commonly observed when biological molecules are used in QDs synthesis. ${ }^{15,16}$ Despite the differences in size, NPs quantum yield was similar for both solutions (15.8 and $14.1 \%$ for green and red NPs, respectively).

Analyses of the crystal structures of quantum dot nanocrystals are scarce and, to date, only two have been described. ${ }^{21-25}$ The first is a cubic metastable phase that belongs to the $F 4 \overline{3} m$ space group, and the second is a hexagonal phase that belongs to the $P 6_{3} m c$ space group (known as $\alpha$-CdS). In a previous study, CdS NPs were synthesized through a mechanochemical method, finding a mixture between both phases (Tolia et al., 2012). By analysing the cubic phase by X-ray diffraction and using the Scherrer formula, a lattice parameter of the phase equal to $0.577 \mathrm{~nm}$ was obtained. Favero et al., 2006 performed first-principle calculations for bulk CdS and found $a=0.588 \mathrm{~nm}$ value for the cubic phase and $a=0.411 \mathrm{~nm}$ and $c=0.674 \mathrm{~nm}$ values for the hexagonal phase. To identify the crystal structure of biomimetic NPs, we analysed a blue-green solution by TEM and HR-TEM. The ring diffraction pattern (RDP) of the synthesized NPs could be indexed considering the cubic phase (Fig. 3a). Further, HR-TEM analysis of Fig. $3 \mathrm{~b}$ showed a NP along $\left[\begin{array}{lll}1 & 0 & 0\end{array}\right]$ the zone axis and the Fourier transform of the image is shown in the inset. The spots indexed as

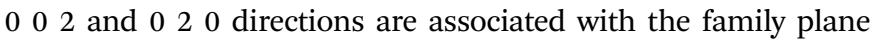
displaying an interplanar distance of $0.29 \pm 0.01 \mathrm{~nm}$, in agreement with the direct measurements performed by HR-TEM. Another HR-TEM image of a NP is shown in Fig. 3C, in this

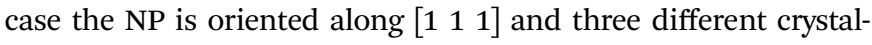
lographic planes can be identified, each one oriented sixty degrees with respect to the other. Interplanar distances of $0.21 \pm 0.01 \mathrm{~nm}$ were determined for the three cases. These results were confirmed by the Fourier transform shown in the inset of the figure, where the different vectors were indexed as 220 . Together, our results show that NPs produced by the biomimetic method described herein are cubic phase CdS nanocrystals.

The chemical nature of the produced NPs was evaluated by FT-IR spectroscopy (Fig. 3C). Signals for Pi and MSA were observed, suggesting that NPs have Pi and thiol in their shell. For $\mathrm{Pi}$, the signals corresponding to the phosphate group $\mathrm{PO}_{4}{ }^{3-}$ $\left(1085 \mathrm{~cm}^{-1}\right)$ and the $\mathrm{P}-\mathrm{O}-\mathrm{P}$ stretching $\left(996 \mathrm{~cm}^{-1}\right)$ were found. For MSA, the signals for the $\mathrm{CH}$ and $\mathrm{CH}_{2}$ stretching (between 3000 and $\left.1800 \mathrm{~cm}^{-1}\right), \mathrm{C}-\mathrm{S}$ stretching $\left(1390 \mathrm{~cm}^{-1}\right)$, and the shift from $\mathrm{COOH}$ (pure MSA) $\rightarrow \mathrm{COO}^{-}$(NPs) $\left(1700 \rightarrow 1563 \mathrm{~cm}^{-1}\right)$ were observed. Previous studies also reported signals for the C-S stretching and the shift from $\mathrm{COOH}$ (pure MSA) $\rightarrow \mathrm{COO}^{-}$ (NPs) in water soluble-CdS QDs capped with mercaptopropionic acid. ${ }^{4}$ Moreover, S-H stretching of MSA (2569 and $2566 \mathrm{~cm}^{-1}$ ) disappeared in the NPs sample, suggesting the presence of a covalent bond between the thiol and the NP through the sulfhydryl group. Finally, a robust $\mathrm{O}-\mathrm{H}$ stretching band is observed $\left(3400 \mathrm{~cm}^{-1}\right)$, suggesting the presence of water molecules at the surface of NPs, as previously reported for water soluble CdS QDs., ${ }^{\mathbf{4 2 0 , 2 6 , 2 7}}$

In summation, the results confirm that our Pi-dependent biomimetic approach provides a simple, clean and safe method
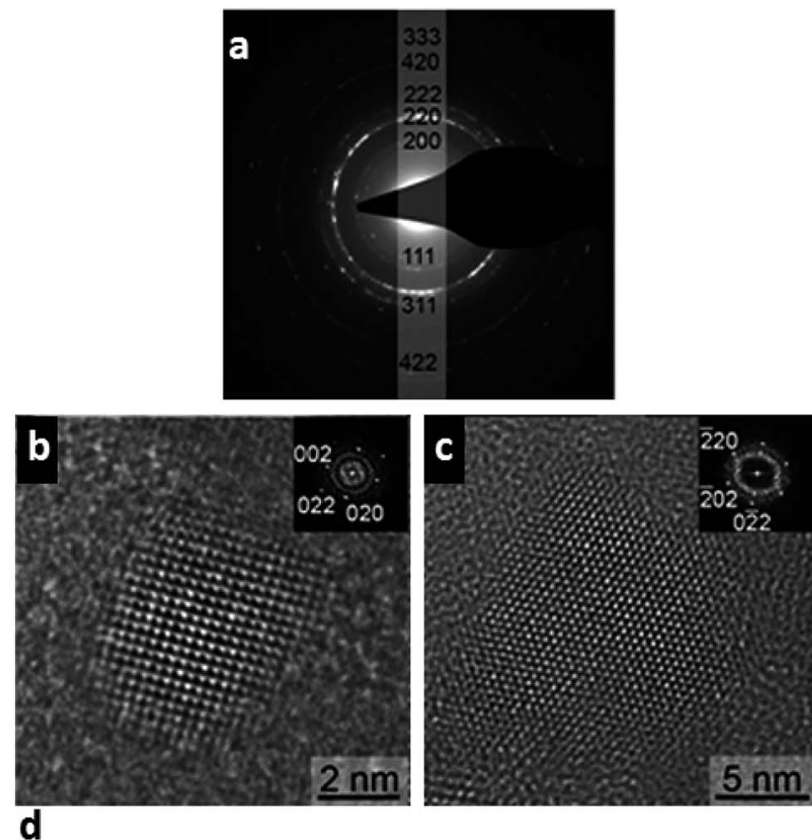

d
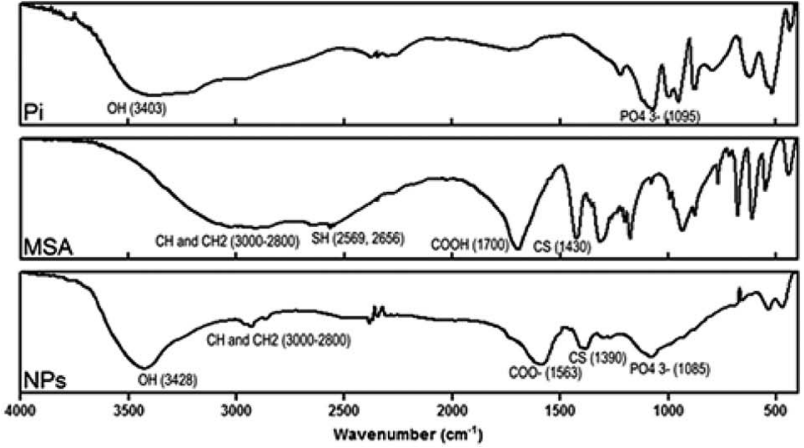

Fig. 3 Structural analysis of CdS QDs. (a) Ring diffraction patterns of biomimetic NPs. (b) and (c) HR-TEM image of particles oriented along the zone axis [ [ $\left.\begin{array}{lll}1 & 0 & 0\end{array}\right]$ for (b) and [ $\left.\begin{array}{lll}1 & 1 & 1\end{array}\right]$ for (c). The indexed Fourier transform are shown in the insets. And (d) shows an FT-IR spectrum of biomimetic CdS QDs. From top to the bottom; Pi, MSA and orange-red NPs. Characteristic signals of each compound are shown in each graph.

to produce water soluble-CdS QDs at low temperatures, physiological $\mathrm{pH}$ and aerobic conditions. To the best of our knowledge, this is one of the simplest methods described to date. Based on our results, we decided to explore if other phosphate sources could also allow the production of NPs. We focused on biological phosphorylated molecules for several reasons: first, as a way of strengthening our green biomimetic method; second, to provide insights into the mechanism of the biological synthesis of QDs; and finally, to identify novel biological targets that could be involved in the cellular interactions of QDs and/or the metals of which they are composed (cadmium in this case).

\section{Biological phosphorylated molecules trigger the biomimetic synthesis of CdS QDs}

Several phosphorylated molecules including G6P, G2P, F1,6BP, AMP, ADP and ATP were tested as phosphate sources for NP production. Cd-MSA solutions supplemented with AMP, 
F1,6BP, G6P or G2P, but not ATP or ADP, showed fluorescence of variable colours over time (Fig. 4A), suggesting that CdS QDs were produced.

As proof of principal, we used G6P to demonstrate that phosphorylated molecules can mediate CdS QDs production. For this purpose, the photophysical properties, composition, and size of the generated NPs was evaluated. For green NPs, the absorbance and fluorescence spectra matched the expected pattern for CdS QDs, with an absorbance shoulder between 350 and $400 \mathrm{~nm}$ and a fluorescence emission peak at $500 \mathrm{~nm}$ (Fig. 4B). FT-IR analysis showed the presence of the stretching bands of $\mathrm{CH}$ and $\mathrm{CH}_{2}$ (between 3000 and $1800 \mathrm{~cm}^{-1}$ ) and $\mathrm{C}-\mathrm{S}$ $\left(1390 \mathrm{~cm}^{-1}\right)$, characteristic of MSA. The sulfhydryl group band was not observed, suggesting the presence of an oxidized thiol. Further, the phosphate group signals and the asymmetrical and symmetrical stretching of P-O-C were observed between 1090 and $1100 \mathrm{~cm}^{-1}$ and $\sim 816 \mathrm{~cm}^{-1}$, respectively (Fig. 4C). As expected, the G6P control also displayed a band between 1090 and $1100 \mathrm{~cm}^{-1}$, which corresponds to the asymmetrical and symmetrical $\mathrm{P}-\mathrm{O}-\mathrm{C}$ stretching. Finally, a DLS size analysis shows that green G6P-NPs have an average size of $35 \mathrm{~nm}$ (data not shown). This size is not that expected for single QDs; however, this might be due to aggregation of NPs as has been described previously for water soluble-QDs. ${ }^{28-30}$

Interestingly, metabolic intermediates of the glycolytic pathway, such as G6P and F1,6BP, have not been used before for QDs synthesis. This simple biomimetic protocol could be used to find other phosphorylated molecules that allow the synthesis of novel organic phosphorylated-QDs. Besides, these results
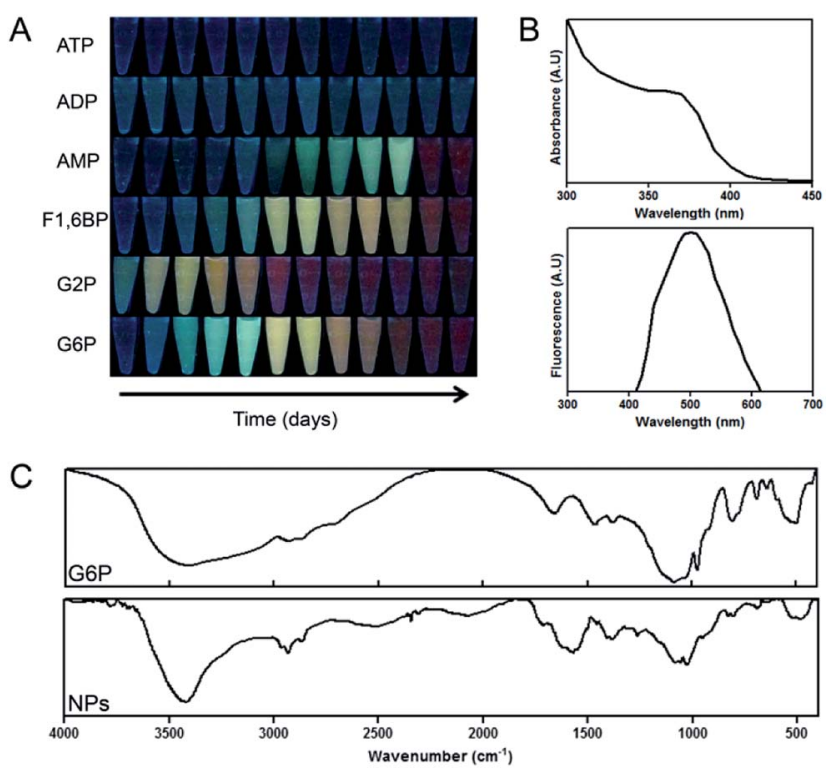

Fig. 4 Biomimetic synthesis of CdS QDs using phosphorylated biomolecules. (A) Fluorescence emission kinetics of different synthesis of CdS QDs in witch $\mathrm{Pi}$ was replaced by different phosphorylated molecules: ATP, ADP, AMP, F1,6BP, G2P and G6P. (B) absorbance and fluorescence (excitation at $350 \mathrm{~nm}$ ) spectra of green NPs purified from a reaction of $5 \mathrm{mM} \mathrm{MSA}, 54.5 \mu \mathrm{M} \mathrm{CdCl}_{2}$ and $10 \mathrm{mM}$ G6P. (C) FT-IR spectrum of G6P and purified green NPs produced in the presence of G6P (G6P-NPs). open a possibility for evaluating new compounds that could participate in biological synthesis of CdS NPs, such as inorganic and/or organic phosphate sources. To date, cellular thiols are the main factor influencing the biosynthesis of sulphide metalQDs, but not other aspects/molecules have been evaluated. ${ }^{16,31}$

Although all tested phosphorylated molecules have in common the presence of at least one phosphate group, the synthetic profile differs among them (Fig. 4A). Dooley et al. described in 2007 that under specific conditions guanosine triphosphate (GTP) was the only nucleotide able to conduct the synthesis of aqueous soluble CdS QDs. The same was observed for PbS QDs using GTP. ${ }^{32}$ This report is in agreement with the results obtained here with ATP and ADP. On the other hand, it has been reported that guanine mono or di-phosphate nucleotides are not as good as GTP for the synthesis of CdS and PbS QDs; ${ }^{33}$ nevertheless, we observed the opposite effect using adenine nucleotides AMP, ADP and ATP; the monophosphate nucleotide was the only capable to synthesize QDs (Fig. 4A). In this context, it is well known that intramolecular coordination produce more stable complexes than coordination through independent ligands, so in the case of ATP and ADP putative formed complexes between Cd and di or tri-phosphate nucleotides present more stability (two coordination sites versus one) than an AMP-Cd complex (at the same conditions) sequestering the metal and competing with the nanocrystalline formation of CdS. Contrarily, the previous referenced study ${ }^{33}$ suggest that phosphate groups on nucleotides are important to control the degree of supersaturation, nucleation and growth of NPs, probably due to their capability to bind metallic atoms. Authors indicate that triphosphates of GTP act in a concerted way with other functional groups of the phosphorylated nucleoside. In fact, GTP molecules with truncated functional groups are unable to bind $\mathrm{Pb}$ ions. Thus, a nitrogen atom from the nitrogen-base is relevant for triggering the synthesis of PbS QDs. ${ }^{32}$

Based on this, we also decided to evaluate if other(s) properties of phosphate, different to its "metal-chelating nature", are important for QDs synthesis.

\section{On the mechanism of Pi-dependent biomimetic synthesis of} CdS QDs

As mentioned before, several protocols to synthesize water soluble-QDs have been developed, but the importance of only one phosphorylated molecule has been suggested to date; the nucleotide GTP (discussed above). In this context, it is important to note that phosphate and the nitrogen atom near the N2chelating of the guanine moiety in GTP are both "good bases". ${ }^{2}$ To evaluate if the effect of $\mathrm{Pi}$ in biomimetic synthesis is consequence of its good base nature, three experiments were performed; in the first one we evaluated the synthesis of QDs using $\mathrm{PB}\left(\mathrm{K}_{2} \mathrm{HPO}_{4} / \mathrm{KH}_{2} \mathrm{PO}_{4}\right), \mathrm{K}_{2} \mathrm{HPO}_{4}$ or $\mathrm{KH}_{2} \mathrm{PO}_{4}$; in the second we used citrate (a good base/chelating molecule) to conduct NPs synthesis, and in the third we tested if triethylamine $\left(\mathrm{Et}_{3} \mathrm{~N}\right)$, a classical good base molecule unable to chelate cations, can trigger NPs synthesis.

$\mathrm{PB}\left(\mathrm{pH}\right.$ 7.4) and $\mathrm{K}_{2} \mathrm{HPO}_{4}(\mathrm{pH}$ 8.9-9.5) trigger the synthesis of QDs; nevertheless, $\mathrm{KH}_{2} \mathrm{PO}_{4}(\mathrm{pH} 3.0-4.0)$ did not presents the 


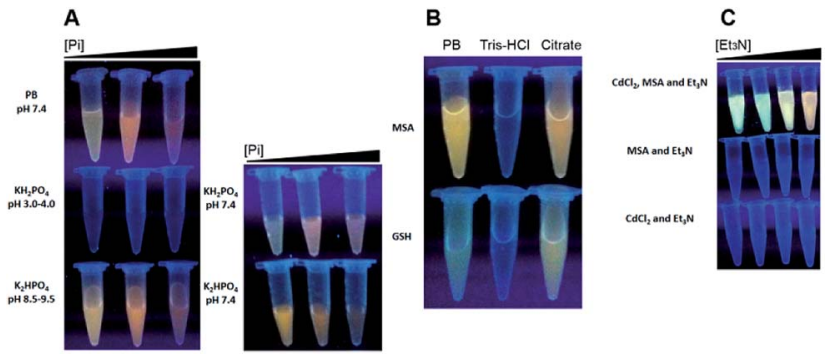

Fig. 5 Effect of $\mathrm{pH}$, citrate and $\mathrm{Et}_{3} \mathrm{~N}$ on biomimetic synthesis of $\mathrm{CdS}$ QDs. (A) Reactions involving $\mathrm{CdCl}_{2} 54.5 \mu \mathrm{M}, \mathrm{MSA} 5 \mathrm{mM}$ and phosphate buffer (PB), $\mathrm{KH}_{2} \mathrm{PO}_{4}$ or $\mathrm{K}_{2} \mathrm{HPO}_{4}$ at their intrinsic $\mathrm{pHs}$ (left) or at $\mathrm{pH} 7.4$ (right). From left to right concentrations of $\mathrm{Pi}$ sources are increased (10, 20 and $40 \mathrm{mM}$ ). (B) Reactions involving $\mathrm{CdCl}_{2} 54.5 \mu \mathrm{M}, \mathrm{MSA} 5 \mathrm{mM}$ and $\mathrm{Et}_{3} \mathrm{~N}(1,2.5,5.0,10$ and $20 \mathrm{mM})$. Control reactions were performed in the absence of $\mathrm{CdCl}_{2}$ (middle) or MSA (down). (C) Reaction involving $\mathrm{CdCl}_{2} 54.5 \mu \mathrm{M}, \mathrm{MSA} 5 \mathrm{mM}$ and citrate $10 \mathrm{mM}$. PB and Tris- $\mathrm{HCl} \mathrm{pH} 7.4$ were used as positive and negative controls, respectively. Fluorescence emission was evaluated after $48 \mathrm{~h}$ incubation at $37^{\circ} \mathrm{C}$.

same effects (Fig. 5A, left). This result supports the hypothesis that the good base nature of $\mathrm{Pi}$ is important for CdS QDs synthesis because $\mathrm{K}_{2} \mathrm{HPO}_{4}$ behaves as a good base at $\mathrm{pH}$ values higher than 7.0, suggesting that the presence of $\mathrm{HPO}_{4}{ }^{2-}$ instead of $\mathrm{H}_{2} \mathrm{PO}_{4}{ }^{-}$is required for Pi-mediated QDs synthesis. In fact, when $\mathrm{H}_{2} \mathrm{PO}_{4}{ }^{-}$(bad base solution) is forced to switch to $\mathrm{HPO}_{4}{ }^{2-}$ (good base solution) by increasing the $\mathrm{pH}$ from 3.0-4.0 to 7.4, the synthesis works properly (Fig. 5A, right). In this context, Tris-HCl buffer is not capable to trigger the synthesis of CdS at pHs higher than 7.0, but citrate does (Fig. 5B). These results suggest that besides the intrinsic $\mathrm{pH}$ driving effect of the synthesis' conducting molecules, the coordination of free $\mathrm{Cd}^{2+}$ atoms could be also an important feature to consider; for example, considering ATP versus AMP complexes (see above), or in this case tris(hydroxymethyl)aminomethane (Tris-buffer molecule) versus citrate or phosphate coordination. However, $\mathrm{Et}_{3} \mathrm{~N}$ also mediates the synthesis of QDs (Fig. 5C), suggesting that the basic characteristic of $\mathrm{Pi}$ ( $\mathrm{pH}$ driving effect) is more important than its chelating capability for QDs synthesis, since $\mathrm{Et}_{3} \mathrm{~N}$ is unable to chelate $\mathrm{Cd}^{2+}$ ions.

On the other hand, another relevant issue for the synthesis of metal sulphide NPs is the necessity of $\mathrm{H}_{2} \mathrm{~S}^{34-36}$ Based on this, $\mathrm{H}_{2} \mathrm{~S}$ required for the biomimetic synthesis should be obtained from CYS, GSH or MSA. We evaluated if $\mathrm{Pi}$ (and $\mathrm{Et}_{3} \mathrm{~N}$ as a control) influences $\mathrm{H}_{2} \mathrm{~S}$ release from thiols under biomimetic conditions.

We determined that $\mathrm{H}_{2} \mathrm{~S}$ release, from the different thiol sources, increases as the concentration of $\mathrm{Pi}$ or $\mathrm{Et}_{3} \mathrm{~N}$ increases (Fig. 6, top graphs). The only exception was MSA and Pi at concentrations above $10 \mathrm{mM}$, in which $\mathrm{H}_{2} \mathrm{~S}$ was not detected (same as negative control).

Interestingly, the amount of $\mathrm{H}_{2} \mathrm{~S}$ detected strongly decreases in the presence of $\mathrm{Cd}$ (data not shown), probably as consequence of CdS nanocrystallization associated to the interaction of $\mathrm{Cd}^{2+}$ and sulphur. Moreover, sulphide production is also observed in the presence of G6P and thiols (Fig. 6, bottom graph). No sulphide release was obtained in presence of

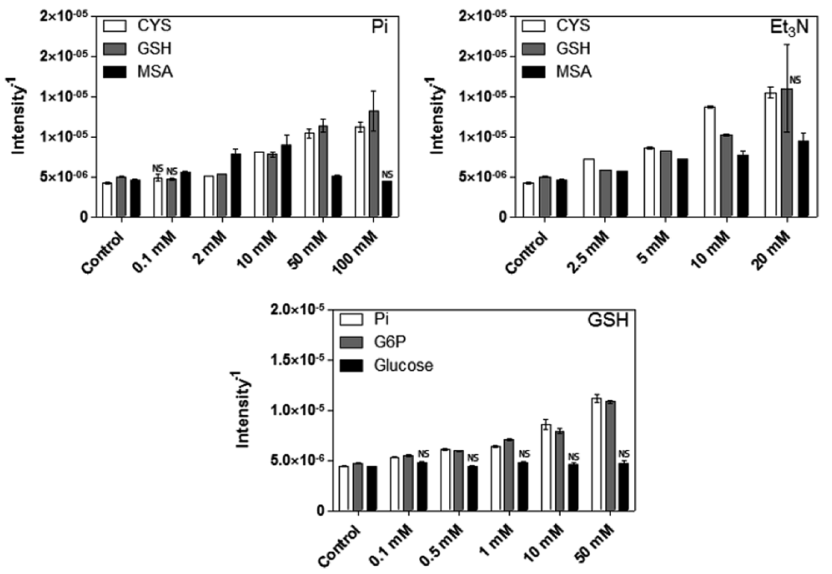

Fig. 6 Phosphate-dependent $\mathrm{H}_{2} \mathrm{~S}$ release from thiols. The generation of $\mathrm{H}_{2} \mathrm{~S}$ was evaluated in different synthesis conditions. For the top graphs, tubes containing $\mathrm{Pi}$ or $\mathrm{Et}_{3} \mathrm{~N} \mathrm{mM}$, and $5 \mathrm{mM} \mathrm{CYS,} \mathrm{GSH}$ or MSA were tested. Bottom graph corresponds to the experiment using Pi, G6P or glucose and GSH 5 mM. Negative controls were prepared using thiols $5 \mathrm{mM}$ in Tris- $\mathrm{HCl} 10 \mathrm{mM}$ (upper) and Pi, G6P or glucose $10 \mathrm{mM}$ (bottom). All solutions were incubated at $37{ }^{\circ} \mathrm{C}$ during $24 \mathrm{~h}$. For statistical analysis, a $P$ value below 0.05 was considerable as significant. Every dataset was compared against its control. No significant (NS) conditions are labelled at the top of the bars, and non-labelled conditions are statistical significant than the controls.

glucose, confirming that the phosphate group contained on G6P molecule is required for $\mathrm{H}_{2} \mathrm{~S}$ release. In conclusion, the importance of phosphates in CdS QDs synthesis is related, at least in part, with the release of $\mathrm{H}_{2} \mathrm{~S}$ from thiols as consequence of its basic characteristics.

$\mathrm{H}_{2} \mathrm{~S}$ gas release from thiols can occur through different mechanisms, being the most common the temperaturepromoted decomposition. For instance, CYS and GSH release $\mathrm{H}_{2} \mathrm{~S}$ during thermal treatment at $110{ }^{\circ} \mathrm{C}^{37}$ Besides, eukaryote and prokaryote organisms can release $\mathrm{H}_{2} \mathrm{~S}$ enzymatically. ${ }^{38}$

Nevertheless, no studies that directly relate - especially organic-phosphates with the generation of $\mathrm{H}_{2} \mathrm{~S}$ from thiols have been reported. Some indirect evidences relating $\mathrm{H}_{2} \mathrm{~S}$ generation from biological thiols and phosphate have been published. $\mathrm{H}_{2} \mathrm{~S}$ is produced from cysteine activated hydrogen sulphide donors in the presence of PBS. ${ }^{39}$ Similar results were obtained recently using chemical thiols that produce sulphide in the presence of PBS. ${ }^{40}$ Finally, a modified-diclofenac molecule with an $\mathrm{H}_{2} \mathrm{~S}$ releasing moiety on it, produces sulphide in presence of a rat liver homogenate dissolved on PBS buffer. ${ }^{41}$ Surprisingly, all these studies use PBS only as a buffer, but do not discuss a possible participation of phosphates on $\mathrm{H}_{2} \mathrm{~S}$ production.

\section{Inorganic phosphate controls the bacterial synthesis of CdS QDs}

Previously, we used a genetically modified $E$. coli strain that overexpressed gshA (involved in GSH synthesis) to synthesize CdS QDs. This strain had increased GSH levels allowing intracellular CdS NPs synthesis in cells exposed to a Cd salt. ${ }^{16}$ Based on the results from the biomimetic method, we hypothesized that Pi could favour CdS NPs biosynthesis in a not genetically 
modified E. coli strain by increasing the basal levels of thiols. To test this, we varied Pi concentrations and evaluated CdS QDs production in wild type $E$. coli. The results showed that; (i) in the absence of Cd or Pi fluorescence was not detected, (ii) at a fixed Cd concentration, cells turned fluorescent in a Pi-dependent way; green at low $\mathrm{Pi}$ concentrations (1 to $2.5 \mathrm{mM}$ ), yellow between 5 and $20 \mathrm{mM} \mathrm{Pi}$, and red at high Pi concentrations (40 to $50 \mathrm{mM}$ ), and (iii) when Pi was not added to cultures exposed to $\mathrm{Cd}$, fluorescence was similar to untreated control cells (Fig. 7A). We also evaluated changes in the colour of cell fluorescence over time. Cells treated with $\mathrm{Cd}^{2+}$ in the absence of Pi did not change, while the fluorescence of cells treated with $\mathrm{Cd}$ and $\mathrm{Pi}$ (50 mM, Fig. 7A) changed from green $(2 \mathrm{~h})$, to yellow ( $8 \mathrm{~h})$ and finally to orange at $24 \mathrm{~h}$ (Fig. 7B). This change in colour is typical of QDs as previously described using different bacterial species. ${ }^{\mathbf{1 6 , 3 0 , 4 2}}$

Regarding the intrinsic characteristics of QDs, the possibility of controlling the fluorescence colour and hence NPs size, remains understudied in microbial biosynthetic protocols already published. ${ }^{\mathbf{1 6 , 4 2 - 5 1}}$ One study suggests CdS NPs size can be "tuned" when using an engineered Stenotrophomonas maltophilia strain that produces extracellular NPs associated with cystathionine gamma-lyase; which would conduct NPs biosynthesis producing $\mathrm{H}_{2} \mathrm{~S}$ from CYS. ${ }^{52}$ In this context, herein we show for the first time that the fluorescence of QDs biosynthesized in a wild type strain can be achieved by modulating the concentration of $\mathrm{Pi}$. These findings represent an alternative to developing improved methods for NPs biosynthesis for which genetic tools are not available, and also represents a possibility to improve bioremediation strategies (towards QDs production for example) for which using genetically modified organisms is sometimes restricted to their application in situ.

Because in the "intracellular" biosynthesis of CdS QDs the divalent cation must first be transported inside cells, Pi could play an important role in this process as it is transported across the cell membrane as a neutral complex $\mathrm{MeHPO}_{4}(\mathrm{Me}=\mathrm{Mg}, \mathrm{Ca}$, Co or $\mathrm{Mn}$ ), through specific transporters called Pit (PitA and PitB). ${ }^{53}$ Pit transporters are also implicated in $\mathrm{Cd}^{2+}$ and $\mathrm{Zn}^{2+}$ uptake in E. coli. ${ }^{54}$ Based on this, we tested if Pi concentration

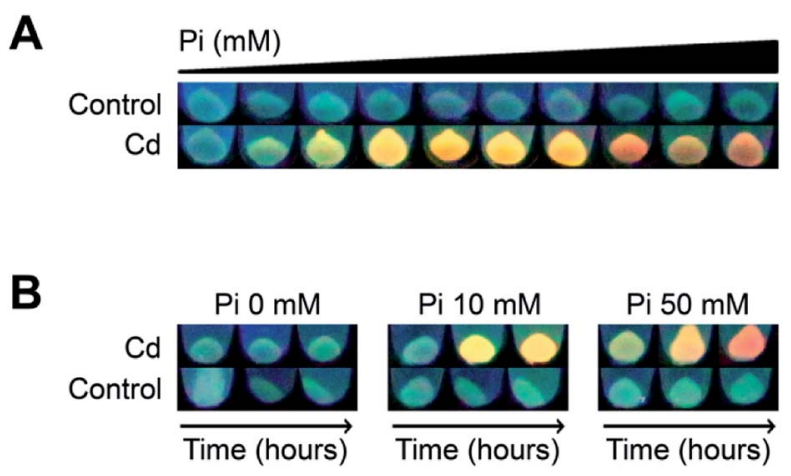

Fig. 7 Pi-dependent biosynthesis of CdS QDs by E. coli. (A) Fluorescence emission of bacterial pellets obtained from cells exposed to $\mathrm{CdCl}_{2}$ and $\mathrm{Pi}$ during $24 \mathrm{~h}$. (B) Fluorescence of bacterial cell pellets exposed to different amounts of Pi during 2, 8 or $24 \mathrm{~h}$. Controls do not contain $\mathrm{Cd}$.
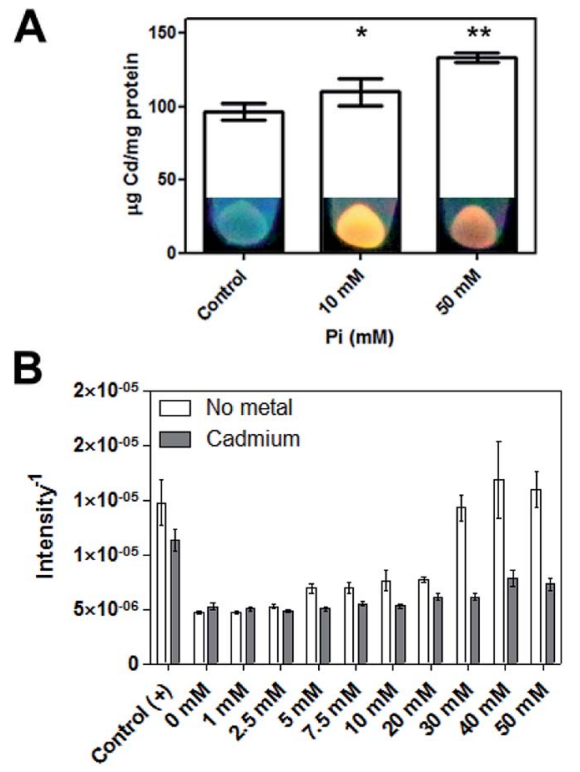

Fig. 8 Effect of $\mathrm{Pi}$ on $\mathrm{Cd}$ content and $\mathrm{H}_{2} \mathrm{~S}$ release of $E$. coli cells biosynthesizing CdS QDs. (A) FAAS quantification of $\mathrm{Cd}$ inside cells exposed to $327.2 \mu \mathrm{M} \mathrm{CdCl}_{2}\left(60 \mu \mathrm{g} \mathrm{mL}^{-1}\right)$ during $24 \mathrm{~h}$, in absence (control) and presence of 10 or $50 \mathrm{mM} \mathrm{Pi}$. Both treatments were compared with the control by $t$-test $(*)=p<0.05$ and $(* *)=p<$ 0.0005 . (B) $\mathrm{H}_{2} \mathrm{~S}$ production in bacterial pellets of cells exposed (or not) to $327.2 \mu \mathrm{M} \mathrm{CdCl}_{2}\left(60 \mu \mathrm{g} \mathrm{mL}^{-1}\right)$ during $24 \mathrm{~h}$, in presence of $\mathrm{Pi}(0 ; 1 ; 2.5$; $5 ; 7.5 ; 10 ; 20 ; 30 ; 40$ and $50 \mathrm{mM}$ ). A bacterial pellet obtained from cells exposed to $327.2 \mu \mathrm{M} \mathrm{CdCl}_{2}$ and $0.5 \mathrm{mM}$ CYS was used as positive control.

had an effect on the intracellular accumulation of $\mathrm{Cd}$. The results showed that $\mathrm{Pi}$ stimulates $\mathrm{Cd}$ accumulation (Fig. 8A). Although $E$. coli can incorporate $\mathrm{Cd}$ in the absence of $\mathrm{Pi}$, the presence of phosphate is required for the intracellular formation of nanocrystals under the tested conditions. Thus, as we determined in the biomimetic synthesis method, Pi is also required for the biosynthesis of CdS QDs in wild type E. coli.

Finally, Pi promoted $\mathrm{H}_{2} \mathrm{~S}$ release from $E$. coli cultures, in agreement with our previous results using the biomimetic method. Even though cells release a basal level of gas in the absence of $\mathrm{Pi}$, when exposed to $\mathrm{Pi}$ in absence of $\mathrm{Cd}, \mathrm{H}_{2} \mathrm{~S}$ release increased as a function of Pi concentration (Fig. 8B). When Pi and $\mathrm{Cd}$ are added simultaneously, less $\mathrm{H}_{2} \mathrm{~S}$ was released, suggesting that $\mathrm{Cd}$ sequestered the gas that was generated by the action of Pi, triggering CdS NPs formation.

Considering all the results presented in this work, we hypothesize that CdS QDs biosynthesis is a process where Cd is sequestered and sulphur and phosphorus metabolisms converge, possibly operating as a detoxifying mechanism. Understanding the biosynthetic process in detail will contribute to developing greener and better methods to produce this nanobiotechnological product of great interest.

\section{Conclusions}

In summation, the results presented herein show that Pi and other biological phosphorylated molecules are implicated in the 
biomimetic synthesis of water soluble-CdS QDs at physiological temperature, and that $\mathrm{Pi}$ favours this process in $E$. coli. The ability of Pi to act as a good base is key issue in triggering $\mathrm{H}_{2} \mathrm{~S}$ production from thiols, which in turn is required to form nanocrystalline CdS by interacting with $\mathrm{Cd}^{2+}$ ions.

\section{Conflicts of interest}

There are no conflicts to declare.

\section{Acknowledgements}

This work was supported by Erika Elcira Donoso López, Fondecyt 1151255 (J.M.P.), INACH RT-25-16 (J.M.P., DB), UNAB DI 488-14/R (J.M.P.), and AFOSR FA9550-15-1-0140 (J.M.P.). A doctoral fellowship from CONICYT to JPM is also acknowledged. FAV thanks Mauricio Cabañas (CIMIS, Universidad Andres Bello) for assistance with DLS measurements, Dr Andrés Vega (Departamento de Ciencias Químicas, Universidad Andres Bello) for his comments and technical support with FT-IR machine and to Dr Mayra Martínez (Chemistry Department, McGill University) for her comments that improved the manuscript.

\section{References}

1 W. R. Algar, A. J. Tavares and U. J. Krull, Anal. Chim. Acta, 2010, 673(1), 1-25, DOI: 10.1016/j.aca.2010.05.026.

2 J. Bang and P. Kamat, ACS Nano, 2009, 3, 1467-1476, DOI: 10.1021/nn900324q.

3 Y. Yin, R. Wang and L. Zhou, Luminescence, 2014, 29(6), 626629, DOI: 10.1002/bio.2595.

4 P. Kumar, D. Kukkar, A. Deep, S. C. Sharma and L. M. Bharadwaj, Adv. Mater. Lett., 2012, 3(6), 471-475, DOI: 10.5185/amlett.2012.icnano.296.

5 M. Fang, C. W. Peng, S. P. Liu, J. P. Yuan and Y. Li, Univ. Sci. Technol., Med. Sci., 2013, 33(4), 520-524, DOI: 10.1007/ s11596.

6 J. Wang, Y. Lu, F. Peng, Y. Zhong, Y. Zhou, X. Jiang, Y. Su and Y. He, Biomaterials, 2013, 34(37), 9509-9518, DOI: 10.1016/ j.biomaterials.2013.09.005.

7 Y. P. Zhang, P. Sun, X. R. Zhang, W. Yang and C. S. Si, Nanoscale Res. Lett., 2013, 8(1), 294, DOI: 10.1186/1556276X-8-294.

8 B. T. Kurien and R. H. Scofield, Methods Mol. Biol., 2009, 536, 557-571, DOI: 10.1007/978-1-59745-542-8_56.

9 P. Wu, T. Zhao, S. Wang and X. Hou, Nanoscale, 2013, 6(1), 43-64, DOI: 10.1039/c3nr04628a.

10 V. Durán-Toro, A. Gran-Scheuch, N. Órdenes-Aenishanslins, J. P. Monrás, L. A. Saona, F. A. Venegas, T. G. Chasteen, D. Bravo and J. M. Pérez-Donoso, Anal. Biochem., 2004, 450, 30-36, DOI: 10.1016/j.ab.2014.01.001.

11 E. Ying, D. Li, S. Guo, S. Dong and J. Wang, PLoS One, 2008, 3(5), e2222, DOI: 10.1371/journal.pone.0002222.

12 J. Tian, R. Liu, Y. Zhao, Q. Xu and S. Zhao, J. Colloid Interface Sci., 2009, 336(2), 504-509, DOI: 10.1016/j.jcis.2009.04.064.

13 K. N. Thakkar, S. S. Mhatre and R. Y. Parikh, Nanomedicine, 2010, 6(2), 257-262, DOI: 10.1016/j.nano.2009.07.002.
14 N. I. Hulkoti and T. C. Taranath, Colloids Surf., B, 2014, 1, 474-483, DOI: 10.1016/j.colsurfb.2014.05.027.

15 J. M. Pérez-Donoso, J. P. Monrás, D. Bravo, A. Aguirre, A. F. Quest, I. O. Osorio-Román, R. F. Aroca, T. G. Chasteen and C. C. Vásquez, PLoS One, 2012, 7(1), e30741, DOI: 10.1371/journal.pone.0030741.

16 J. P. Monrás, V. Díaz, D. Bravo, R. A. Montes, T. G. Chasteen, I. O. Osorio-Román, C. C. Vásquez and J. M. Pérez-Donoso, PLoS One, 2013, 7(11), e48657, DOI: 10.1371/ journal.pone.0048657.

17 R. Narayanaswamy and F. Sevilla III, Fresenius' J. Anal. Chem., 1988, 329(7), 789-792.

18 A. P. Alivisatos, Science, 1996, 271(5251), 933-937, DOI: 10.1126/science.271.5251.933.

19 Z. H. Zhang, W. S. Chin and J. J. Vittal, J. Phys. Chem. B, 2004, 108, 18569-18574, DOI: 10.1021/jp0470849.

20 J. Tolia, M. Chakraborty and Z. V. P. Murthy, Cryst. Res. Technol., 2012, 47(8), 909-916, DOI: 10.1002/crat.201200077.

21 R. C. Sharma and Y. A. Chang, J. Phase Equilib., 1996, 17(5), 425-431, DOI: $10.1007 /$ BF02667635.

22 R. Sanghi and P. Verma, Chem. Eng. J., 2009, 155(3), 886-891, DOI: 10.1016/j.cej.2009.08.006.

23 K. Prasad and A. K. Jha, J. Colloid Interface Sci., 2010, 342(1), 68-72, DOI: 10.1016/j.jcis.2009.10.003.

24 P. P. Favero, M. Souza-Parise, J. L. R. Fernandez, R. Miotto and A. C. Ferraz, Braz. J. Phys., 2006, 36(3B), 1032-1034.

25 S. Y. Ha, D. S. Yoo, I. G. Kim, M. S. Choo, G. W. Kim, E. S. Lee and B. C. Lee, J. Korean Phys. Soc., 2011, 58(5), 1274-1278, DOI: $10.3938 /$ jkps.58.1274.

26 B. R. Srinivasa, B. R. Kumar, V. R. Reddy and T. S. Rao, Chalcogenide Lett., 2011, 8(3), 177-185.

27 D. Gerion, F. Pinaud, S. C. Williams, W. J. Parak, D. Zanchet, S. Weiss and A. P. Alivisatos, J. Phys. Chem. B, 2001, 105(37), 8861-8871, DOI: 10.1021/jp0105488.

28 Y. Zhang, L. Mi, P. N. Wang, S. J. Lu, J. Y. Chen, J. Guo, W. L. Yang and C. C. Wang, Small, 2008, 4(6), 777-780, DOI: $10.1002 / \mathrm{smll} .200701034$.

29 V. Poderys, M. Matulionyte, A. Selskis and R. Rotomskis, Nanoscale Res. Lett., 2011, 6(1), 9, DOI: 10.1007/s11671-0109740-9.

30 C. Gallardo, J. P. Monrás, D. O. Plaza, B. Collao, L. A. Saona, V. Durán-Toro, F. A. Venegas, C. Soto, G. Ulloa, C. C. Vásquez, D. Bravo and J. M. Pérez-Donoso, J. Biotechnol., 2013, 187, 108-115, DOI: 10.1016/ j.jbiotec.2014.07.017.

31 S. Hinds, B. J. Taft, L. Levina, V. Sukhovatkin, C. J. Dooley, M. D. Roy, D. D. Macneil, E. H. Sargent and S. O. Kelley, J. Am. Chem. Soc., 2006, 128(1), 64-65, DOI: 10.1021/ja057002+.

32 N. Ma, E. H. Sargent and S. O. Kelley, Nat. Nanotechnol., 2009, 4(2), 121-125, DOI: 10.1038/nnano.2008.373.

33 M. Nogami and N. Nagasaka, J. Non-Cryst. Solids, 1992, 147\&148, 331-334, DOI: 10.1016/S0022-3093(05)80639-8.

34 S. Gorer, J. A. Ganske, J. C. Hemminger and R. M. Penner, J. Am. Chem. Soc., 1998, 120(37), 9584-9593, DOI: 10.1021/ ja981676l. 
35 A. S. Deshpande, R. B. Khomane, B. K. Vaidya, R. M. Joshi, A. S. Harle and B. D. Kulkarni, Nanoscale Res. Lett., 2008, 3(6), 221-229, DOI: 10.1007/s11671-008-9140-6.

36 Y. Zheng and C. T. Ho, ACS Symp. Ser., 1994, 11, 138-146, DOI: 10.1021/bk-1994-0564.ch011.

37 Y. Zhao, T. D. Biggs and M. Xian, Chem. Commun., 2014, 50, 11788-11805, DOI: 10.1039/C4CC00968A.

38 Y. Zhao, H. Wang and M. Xian, J. Am. Chem. Soc., 2012, 133(1), 15-17, DOI: 10.1021/ja1085723.

39 A. Martelli, L. Testai, V. Citi, A. Marino, I. Pugliesi, E. Barresi, G. Nesi, S. Rapposelli, S. Taliani, F. Da Settimo, M. C. Breschi and V. Calderone, ACS Med. Chem. Lett., 2013, 4(10), 904908, DOI: $10.1021 / \mathrm{ml} 400239$ a.

40 J. L. Wallace, G. Caliendo, V. Santagada, G. Cirino and S. Fiorucci, Gastroenterology, 2007, 132(1), 261-271, DOI: 10.1053/j.gastro.2006.11.042.

41 C. T. Dameron, R. N. Reese, R. K. Mehra, A. R. Kortan, P. J. Carroll, M. L. Steigerwald, L. E. Brus and D. R. Winge, Nature, 1989, 338, 596-597, DOI: 10.1038/338596a0.

42 G. Ulloa, B. Collao, M. Araneda, B. Escobar, S. Álvarez, D. Bravo and J. M. Pérez-Donoso, Enzyme Microb. Technol, 2016, 95, 217-224.

43 D. Plaza, C. Gallardo, Y. Straub, D. Bravo and J. M. PérezDonoso, Microb. Cell Fact., 2016, 15(76), DOI: 10.1186/ s12934-016-0477-8.

44 J. D. Holmes, D. J. Richardson, S. Saed, R. Evans-Gowing, D. A. Russell and J. R. Sodeau, Microbiol., 1997, 143(8), 2521-2530, DOI: 10.1099/00221287-143-8-2521.
45 R. Y. Sweeney, C. Mao, X. Gao, J. L. Burt, A. M. Belcher, G. Georgiou and B. L. Iverson, Chem. Biol., 2014, 11, 15531559, DOI: 10.1016/j.chembiol.2004.08.022.

46 H. J. Bai, Z. M. Zhang, Y. Guo and W. Jia, Nanoscale Res. Lett., 2009, 4(7), 717-723, DOI: 10.1007/s11671-009-9303-0.

47 H. J. Bai, Z. M. Zhang, Y. Guo and G. E. Yang, Colloids Surf., $B$, 2009, 70(1), 142-146, DOI: 10.1016/j.colsurfb.2008.12.025.

48 S. H. Kang, S. Singh, J. Y. Kim, W. Lee, A. Mulchandani and W. Chen, Appl. Environ. Microbiol., 2007, 73(19), 6317-6320, DOI: 10.1128/AEM.01237-07.

49 S. H. Kang, K. Bozhilov, N. Myung, A. Mulchandani and W. Chen, Angew. Chem., Int. Ed. Engl., 2008, 47, 5186-5189, DOI: 10.1002 /anie.200705806.

50 T. J. Park, S. Y. Lee, N. S. Heo and T. S. Seo, Angew. Chem., Int. Ed. Engl., 2010, 49(39), 7019-7024, DOI: 10.1002/ anie. 201001524.

51 A. Syed and A. Ahmad, Spectrochim. Acta, Part A, 2013, 106, 41-47, DOI: 10.1016/j.saa.2013.01.002.

52 Z. Yang, L. Lu, V. F. Berard, Q. He, C. J. Kiely, B. W. Berger and S. McIntosh, Green Chem., 2015, 17, 3775, DOI: 10.1039/c5gc00194c.

53 H. W. van Veen, T. Abee, G. J. Kortstee, W. N. Konings and A. J. Zehnder, Biochemistry, 1994, 33(7), 1766-1770, DOI: 10.1021/bi00173a020.

54 S. J. Beard, R. Hashim, G. Wu, M. R. B. Binet, M. N. Hughes and R. K. Poole, FEMS Microbiol. Lett., 2006, 184(2), 15746968, DOI: 10.1111/j.1574-6968.2000.tb09019.x. 